\title{
Lensless focusing with subwavelength resolution by an array of nanoholes
}

\author{
Fu Min Huang ${ }^{1}$, Yifang Chen ${ }^{2}$, F. Javier Garcia de Abajo ${ }^{3}$, Nikolay Zheludev ${ }^{1}$ \\ 1. EPSRC Nanophotonics Portfolio Centre, Optoelectronics Research Centre, University of Southampton, SO17 1BJ, \\ $U K$ \\ 2. Central Microstructure facility, Rutherford Appleton Laboratory, Didcot, Oxon, OX11 OQX, UK \\ 3. Instituto de Optica, CSIC, Serrano 121, 28006 Madrid, Spain
}

We provide the first experimental evidence of focusing light into subwavelength spots by a 2-dimensional planar photonic structure. When a quasi-periodic array of nanoholes was illuminated with coherent light sources, it created very rich diffraction patterns at different heights to the array. At specific heights, we observed diffraction patterns containing subwavelength spots. The ability to focus light into subwavelength spots is of fundamental importance for applications in a wide range of areas, e.g. microscopy, photo lithography, data storage etc.
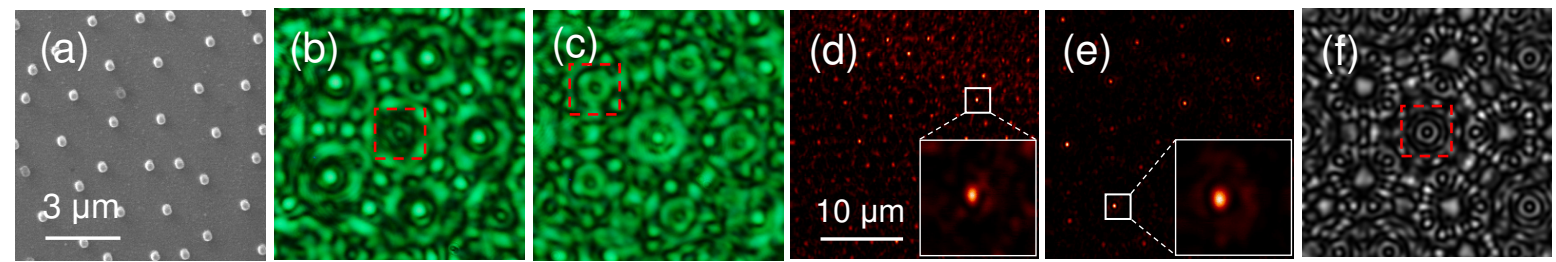

Fig.1 (a) SEM image of a fragment of a quasi-periodic nanohole array, which show a Penrose-like pattern. The minimum distance between two neighbouring holes is $1.2 \mu \mathrm{m}$. Photographs of the diffraction patterns at height $h=7.1 \mu \mathrm{m}$ (b) and $h=8.5 \mu \mathrm{m}$ (c), when illuminated by a coherent laser with $\lambda=500 \mathrm{~nm}$. Two examples of subwavelength spots with FWHM of $210 \mathrm{~nm}$ (b) and $170 \mathrm{~nm}$ (c) are highlighted. SNOM images of the diffraction patterns measured at height $h=5 \mu \mathrm{m}(\mathrm{d})$ and $h=12.5 \mu \mathrm{m}(\mathrm{e})$, when illuminated with $\lambda=660 \mathrm{~nm}$. Subwavelength hot spots are highlighted and zoomed in the insets, with FWHM of $235 \mathrm{~nm}$ and $320 \mathrm{~nm}$, respectively. (f) Calculated diffraction pattern at $h=7.1 \mu \mathrm{m}$ when illuminated with $\lambda=500 \mathrm{~nm}$, which is qualitatively in agreement with the measured diffraction pattern shown in (b). The size of images (a, b, c, f) is $10 \mu \mathrm{m} \times 10 \mu \mathrm{m}$, that of images (d, e) is $30 \mu \mathrm{m} \times 30 \mu \mathrm{m}$, and that of inset images in (d) and (e) is $3 \mu \mathrm{m} \times 3 \mu \mathrm{m}$.

We used a Penrose-like quasi-periodic array of nanoholes with approximate 10 -fold symmetry. It contains about 14,000 holes of $200 \mathrm{~nm}$ in diameter. The sample was manufactured by electron beam lithography in a $100 \mathrm{~nm}$ aluminium film deposited on a silica substrate. A fragment of the SEM image of the sample surface is shown in Fig.1(a). A coherent laser source of $500 \mathrm{~nm}$ illuminated the sample surface from the substrate side. Optical diffraction patterns at different heights to the array were imaged by a digital camera (Canon EOS-350D) through a combined optical system composed of a high numerical aperture objective lens (N.A. 0.95, 150X) working in air and another lens with a magnification of 2.5. Very rich diffraction patterns were observed at different heights to the array. However, at some specific heights we observed patterns with subwavelength spots (Fig.1(b-c)). The FWHM of the central small spot in Fig.1(b) is $\sim 210 \mathrm{~nm}(0.42 \lambda, \lambda=500 \mathrm{~nm})$ and that highlighted in Fig.1(d) is $\sim 170 \mathrm{~nm}(0.34 \lambda)$, which are much smaller than the Rayleigh resolution limit of the microscope $(320 \mathrm{~nm}, 0.64 \lambda)$. In Fig.1(d-e) we show the diffraction patterns at heights of $h=5 \mu \mathrm{m}$ and $h=12.5 \mu \mathrm{m}$ measured by a scanning near field optical microscope (SNOM) with a metal-coated probe of about $120 \mathrm{~nm}$ aperture in diameter, when illuminated by a diode laser of $660 \mathrm{~nm}$. Subwavelength spots are evidently seen. Two of them are highlighted and zoomed (shown in the insets), which indicate a FWHM of $235 \mathrm{~nm}(0.35 \lambda)$ and $320 \mathrm{~nm}(0.48 \lambda)$ across the horizon axis, respectively.

The subwavelength spots were observed either with a conventional optical microscope or a scanning near-field optical microscope at distances of a few tens of wavelengths away from the array, therefore there is little contribution from evanescent waves. Generally it was believed that without evanescent waves, light can not form subwavelength spots. However, as it was found recently [1], functions with limited spectrum can oscillate much faster than the highest frequency Fourier components they contain, a phenomenon called superoscillations. In optics this implies that diffraction of light on certain object can actually form arbitrarily small spots without evanescent waves. To confirm that this quasi-periodic structure can form subwavelength spots without evanescent waves, we calculated the diffraction pattern at $h=7.1 \mu \mathrm{m}(\lambda=500 \mathrm{~nm})$ with only propagating waves based on the diffraction theory of light employing angular spectrum representation of the diffracted field. The result (Fig.1(f)) indeed shows subwavelength spots (the FWHM of the central spot is $160 \mathrm{~nm}$ ), which is qualitatively in agreement with the result of experiments (Fig.1(b)).

\section{References}

1. M. V. Berry and S. Popescu, "Evolution of quantum superoscillations and optical superresolution without evanescent waves”, J. Phys. A: Math.Gen. 39, 6965-6977 (2006). 